\title{
Review \\ Local control by radiotherapy: is that all there is?
} Silvia C Formenti ${ }^{1}$ and Sandra Demaria ${ }^{2}$

\author{
1Sandra and Edward H. Meyer Professor of Radiation Oncology and Medicine, Department of Radiation Oncology, New York University School of \\ Medicine and NYU Cancer Institute, 160 East 34th Street, New York, NY 10016, USA \\ 2Department of Pathology, NYU Cancer Institute, New York University School of Medicine, 550 First Avenue, \\ New York, NY 10016, USA
}

Corresponding author: Silvia C Formenti, silvia.formenti@med.nyu.edu

Published: 5 November 2008

This article is online at http://breast-cancer-research.com/content/10/6/215

(c) 2008 BioMed Central Ltd

Breast Cancer Research 2008, 10:215 (doi:10.1186/bcr2160)

\begin{abstract}
Radiotherapy is a local treatment modality employed in breast cancer to reduce local recurrence following surgery. The observed association of optimal local control with improved survival was not expected in a disease characterized by early systemic spread. The underlying mechanisms whereby the application of ionizing radiation to the primary tumor site can have systemic effects remain unclear and are the subject of much debate. In the present article we discuss the hypothesis that radiotherapy has unique biological effects and that, in addition to killing residual neoplastic cells after surgery is performed, it might favorably alter the microenvironment at the primary tumor site during the process of wound healing and the development of antitumor immune responses.
\end{abstract}

\section{Introduction}

Local control by radiotherapy has been associated with significantly improved breast cancer-specific survival.

The Early Breast Cancer Trialists meta-analysis of the data available from 42,000 breast cancer patients who had participated in 78 contemporary prospective randomized trials (that had started after 1995) has provided invaluable information on the relationship between local control and patients' overall and breast cancer-specific survival [1]. Over a time span of 15 years, for every four local recurrences avoided through optimal application of local treatment, one death from breast cancer could be prevented - with the consequent reduction on 15-year overall mortality. Noticeably the proportional benefit derived by receiving radiotherapy was detected in all patient subsets, independently of patient and tumor characteristics or type of primary surgery (breast conservation versus mastectomy).

This evidence has generated among the breast cancer research community a new wave of reflection on the biology of breast cancer and the effects of available treatment. An example is Dr Punglia and coworkers' editorial in the New
England Journal of Medicine, which engages in a comprehensive analysis to interpret the consequences of local control on breast cancer survival [2]. These authors, however, suggest that achieving optimization of systemic therapy will eventually remove the benefit derived by adding radiotherapy to the management of breast cancer.

We propose a different view, which attributes to radiotherapy unique biological effects capable of directly and specifically interfering with metastasis of breast cancer.

The fact that the benefit occurred only in the context of successful local control suggests that events within the irradiated field are implicated. Until recently, however, the underlying mechanism of this connection remained elusive. A commonly accepted hypothesis is that optimization of local control, by adding radiotherapy to complement surgery, recruits into cure a selected subset of patients who would have otherwise recurred locally (failed surgery) and developed subsequent metastases. This explanation implies a process with distinct waves of cell migration and metastases with differing invasive properties, influenced by events that occur at the primary tumor site.

\section{Metastases dissemination and colonization}

The interpretation mentioned previously is consistent with the emerging biological evidence that the metastatic process is characterized by two distinct phases. The first phase, limited to tumor cell dissemination to distant organs, consists of a successful geographical migration of viable cells far away from the primary tumor [3-5].

To enable migration, cancer cells of epithelial origin need to acquire morphological and functional features identical to those required to heal a wound - a process described as the epithelial to mesenchymal transition (EMT) [6].

$\mathrm{EMT}=$ epithelial to mesenchymal transition; H\&E = hematoxylin and eosin. 
A physiological component of wound repair, the process of EMT is a stromal cell-driven mechanism that conveys motility to epithelial cells to become invasive and permeate the wound site in order to heal the gap created after surgical removal. The same signaling transduction pathways of EMT occur during gastrulation, at the beginning of embryogenesis, when cells transfer from the neural crest of the ectoderm to form the mesoderm, in preparation of mesenchymal tissues for organogenesis. During EMT epithelial cells acquire both the morphology and functional characteristic of fibroblasts. Gene expression shifts from cytokeratins to vimentin, a filament characteristic of the mesenchymal cytoskeleton, while fibronectin, an extracellular matrix protein commonly secreted by fibroblasts, is produced. E-cadherin production is substituted by $\mathrm{N}$-cadherin production, favoring motility of cells [7].

The same complex cellular programs enabling cell migration and invasion during gastrulation in embryogenesis and the physiological repair of a wound are evoked in cancer metastasis, suggesting that the pre-existing genetic program underlying EMT in the cancer cell is strategically reactivated at a crucial point in the natural history of cancer.

Preclinical data have shown that, despite the many challenges circulating tumor cells encounter, early distant dissemination is common even among invasive breast cancers measuring less than $1 \mathrm{~cm}$ in diameter. This process results in micro-metastatic, self-contained foci of cancer cells that persist clinically silent in multiple organs until one of these clusters acquires the necessary properties to establish invasive, colonizing growth, to become a detectable metastatic site and to re-disseminate systemically with a second wave of micro-metastasis. Genetic analysis experiments suggest that the latter step, colonization, is a more selective one, and it is likely to be the step that drives cancer-specific mortality [8].

In this model, the benefit from optimal local control is either derived from interfering with the phase of dissemination, from interfering with that of colonization, or from interfering with both phases. To this end, several rapidly emerging lines of evidence are shedding some light on what until now has consistently eluded a biological explanation.

\section{The surgical cavity as the site for EMT}

Crucial biological effects associated with surgery at the tumor site might impact on breast cancer systemic recurrence. In a recent clinical study of patients with HER2neu overexpressing tumors requiring re-excision to assure negative margins, cell proliferation of the re-excised specimen was higher than that of the original tumor. Besides, fluid harvested from axillary drainage stimulated HER2positive mammary carcinoma cell growth in a two-dimensional growth assay. The effect was abrogated by pretreatment with trastuzumab, suggesting a role for HER2neu in the inflammatory microenvironment that stimulates cell growth at the wound site $[9,10]$.

The original evidence that surgery modifies the kinetics of breast cancer micro-metastasis $[11,12]$ has been recently corroborated by the increasingly recognized analogies of EMT in wound repair and the acquisition of colonization features. At the site of lumpectomy the physiological wound repair process stimulates EMT for the breast tissue to heal. EMT conveys motility to the epithelial cells on the periphery of the cavity to become invasive and move towards the core of the wound to heal the breast after surgical removal. During this phase any residual neoplastic cell at the excision site is also exposed to the physiological signaling associated with inflammation and wound repair. This exposure could conceivably enable neoplastic cells to acquire the tools for EMT, harnessing them to display a more malignant, colonizing behavior.

Evidence in support of this possibility emerged from the work of Nuyten and colleagues, who compared three previously established gene expression profiles of tumor tissue to assess their predictive value for local recurrence after breastconserving surgery [13]. The 70-gene prognosis profile, the hypoxia-induced profile and the wound-response signature were tested in a cohort of 161 patients. At supervised analysis the wound response signature was the only profile with the ability to predict local recurrence: an activated wound pattern versus a nonactivated wound pattern was associated with an increased local recurrence rate. When assessed in a Cox regression model that included classical risk factors for local recurrence (age, tumor diameter and radiotherapy dose to the tumor cavity), the supervised wound response signature added significant prognostic information at multivariable analysis (hazard ratio $=16,95 \%$ confidence interval $=1.9$ to $125, P=0.01)[13]$.

It is also conceivable that breast cancer patients undergoing wound repair at the tumor cavity site generated by surgical excision of the primary tumor could experience a wave of metastatic reseeding/colonization. Interestingly, evidence exists that breast cancer wound complications are associated with an increased risk of systemic recurrence of breast cancer [14].

Postoperative radiotherapy may act by eliminating residual local tumor cells (at the original tumor bed, in the index breast), including those that have undergone EMT changes induced by the microenvironment of wound repair. Other effects of radiotherapy could result in preventing or reducing colonization after EMT. A recent study compared the functional and proteomic characteristics of fluid obtained from the breast cancer patients' post-excisional cavities. The day after surgery, fluid from the wound drainage was collected and compared with preoperative plasma in two separate groups of women who had undergone either 
surgery alone or had also received one intraoperative dose of radiotherapy (20 Gy) [15].

When cocultured with breast cancer cell lines, the fluid from the wound drainage stimulated proliferation, migration, and invasion. Cell motility was assessed by chemotaxis and standard Matrigel invasion assay, and by video time-lapse microscopy of cells included in three-dimensional collagen I. The observed effect was abrogated when the fluid from the wound drainage tested was obtained from patients who had also undergone intraoperative radiotherapy. On proteomic analysis, a distinct profile of cytokine expression characterized irradiated versus nonirradiated fluid from the wound drainage. The authors proposed the concept that intraoperative radiotherapy could have an additional series of effects other than classical cell killing, by abrogating some of the detrimental consequences associated with the formation of a surgical wound.

\section{The tumor site as an immunogenic hub}

An important actor at the tumor site is the immune system. Tumor occurrence when recognized by clinical detection is the obvious consequence of failed immune recognition and rejection. Nevertheless, the immune system remains actively involved in every step of cancer progression, including during the phase of dormancy; for instance, $\mathrm{CD}^{+} \mathrm{T}$ cells maintain the dormant status in preclinical cancer models [16]. Moreover, breast cancer patients with cytokeratin-positive cells in the bone marrow also have an associated infiltration of memory T cells [17].

A common mechanism tumors utilize to avoid rejection consists of altering their phenotype to evade immunity, a process defined as immunoediting [18]. Loss of specific antigens or other important recognition molecules occurs during tumor progression and after immunotherapy. Recent data suggest that EMT could be the result of reprogramming in response to immunological pressure [19]. When a tumor cell line generated from a spontaneous tumor of the neutransgenic mouse and expressing the rat neu antigen was injected into nontransgenic mice it was initially rejected by the immune system. Over time, however, neu antigen-loss cell variants that escaped recognition by the immune system emerged. These tumor epithelial cells were found to display morphological features of EMT, were found at microarray analysis to show upregulation of invasion factors, and were demonstrated to have acquired functional invasiveness when tested in an in vitro migration assay. This work provides preclinical evidence that an immune-mediated mechanism could be at the origin of EMT in cancer [19].

Another immune-mediated mechanism shown to enhance tumor progression is mediated by macrophages [20]. Infiltration of the tumor by tumor-associated macrophages correlates with poor prognosis in breast cancer patients [10,21]. A better understanding of this process will enable novel anticancer applications.

\section{Effect of local ionizing radiation on the immunogenic hub}

Important effects of ionizing radiation govern tumor immunogenicity. Radiotherapy-induced cell killing is a form of cell death effectively sensed by the immune system that contributes important elements necessary to recover/induce tumor-specific immunity potentially capable of rejecting the colonizing clonogenic cells.

Our group has tested in the laboratory the hypothesis that radiation-induced cell death and related danger signals are sensed by the immune system and might contribute to the global success of therapy [22-27]. The generation of this hypothesis came from the clinical experience of testing concurrent chemoradiation in patients with locally advanced breast cancer [28-30]. Characterized by already large, locally advanced tumors at the time of initial diagnosis, these patients rarely have detectable metastatic disease. Nevertheless, if left untreated most patients with locally advanced breast cancer die of metastatic progression in 5 years, suggesting a high metastatic potential.

To address the clinical challenge of tumors that often involved the entire breast and were defined inoperable we originally tested a preoperative treatment to enable surgical removal with a negative margin, without the use of a skin flap to close the wound. A moderate dose of local radiotherapy to the involved breast and regional nodes (50 Gy in 28 fractions) was delivered during continuous-infusion 5-fluorouracil chemotherapy. In a pilot trial of 35 originally inoperable women the combined regimen enabled mastectomy with negative margins in all patients $[28,29]$. A pathological response (defined as disappearance of invasive disease or a limited residual of $<10$ microscopic cancer foci) was achieved in one-third of patients and resulted in a $72 \%$ disease-free survival rate at 5 years - an unexpectedly high rate after a very well tolerated approach - with light systemic therapy [31].

This unexpected success made us consider the possibility that cell death induced by combined therapy might have a systemic effect, and may reduce/delay the clinical expression of micro-metastasis. The immune system appeared the best candidate to contribute to the success encountered.

Multiple lines of evidence supporting this research direction have since emerged. A series of preclinical studies designed to test whether local tumor irradiation could promote systemic tumor control was conducted. A mouse breast cancer model in which tumor cells syngeneic to the recipient immunocompetent mouse strain were implanted at two separate sites demonstrated that irradiation of one tumor site resulted in tumor growth inhibition at the other, nonirradiated, site (abscopal effect) [22]. The administration of a growth factor for dendritic cells was required in order for local tumor irradiation to trigger the antitumor response, suggesting that tumor cell death induced by radiation promoted the presen- 
tation of tumor-derived antigens by the dendritic cells, thus acting to generate an in situ vaccine [22]. A similar requirement for boosting the number/function of dendritic cells, which are often impaired in tumor-bearing hosts [32], was shown by other workers in a lung cancer model in which a high local radiation dose to the primary tumor was able to induce an immune-mediated inhibition of the lung metastasis [33].

Further support for the concept that tumor cells killed by radiation are a good source of antigens came from studies in which, after exogenous dendritic cells were injected directly into the irradiated tumor, a successful induction of antitumor immunity was achieved [34,35]. More recently, the identification of two of the molecular signals that determine the immunogenicity of cancer cells killed by radiation - calreticulin and high-mobility group box 1 alarmin protein - has provided the mechanistic proof that the irradiated tumor can become an in situ vaccine $[36,37]$.

To further translate these data to their clinical implications (that is, to investigate the effect of local control by radiotherapy on survival) we employed the 4T1 syngeneic mouse model, which mimics the behavior of an invasive and metastatic breast cancer and is characterized by early systemic dissemination from the primary implantation site. Outgrowth of lung metastases is usually responsible for death of the animals. In this model, local tumor control by radiation was associated with an improved survival only when an antitumor immune response inhibiting metastases was induced, which required administration of antibodies blocking the CTLassociated antigen-4 receptor - an intervention that prevents immune tolerance associated with suboptimal dendritic cell function [25]. The results support the contention that the effects of local radiotherapy on survival in the setting of tumors with early dissemination are likely to depend on the therapy's ability to alter the tumor-host interactions and to promote antitumor immunity.

\section{Radiation and the microenvironment at the immunogenic hub}

With the improved understanding of the complexity of the tumor microenvironment and its crucial role in regulating tumor progression, the cells and extracellular components that are altered by local radiotherapy are undergoing better definition [38].

In addition to its cytocidal effects, ionizing radiation has recently been shown to cause a plethora of changes on both the cancer cells and tumor stroma, critical in determining its therapeutic success. Many of these changes have been proven in experimental systems to impact the ability of the immune system to reject the tumor [27]. In addition to the exposure of calreticulin and the release of high-mobility group box 1 by dying cancer cells mentioned above, radiation also induces the upregulation of cell surface and soluble molecules that play important roles in mediating the interaction of the tumor with the immune system. Radiationinduced upregulation of Fas/CD95 and major histocompatibility complex class I molecules on cancer cells enhances their recognition and elimination by antitumor T cells [39-41]. Similarly, upregulation of an adhesion molecule (VCAM, vascular cell adhesion molecule 1) on tumor-associated endothelia enhances the infiltration of the tumor by immune cells [42].

We have recently shown that radiation markedly enhances the release by human and mouse breast cancer cells of CXCL16, a chemokine known to play an important role in recruitment of effector $\mathrm{T}$ cells to sites of peripheral inflammation (Figure 1). In the 4T1 mouse model, radiation-induced CXCL16 release was critical for efficient recruitment of antitumor $\mathrm{T}$ cells and tumor inhibition following treatment with radiation and CTL-associated antigen 4 blockade [43]. The modulation of chemokines by radiotherapy is thus another mechanism by which the technique regulates the tumor microenvironment and its relationship with the host immune system.

Since success of standard anticancer therapies appears to depend on recruiting contributing immune-mediated effects, a synergy between immunotherapy and standard cancer therapy needs to be pursued. On the other hand, some of the local and systemic effects of radiotherapy have been shown to enhance invasion and metastatic cancer progression [44]. Only clinical trials will provide support to the hypothesis that immune mechanisms underlie the effect of local control on systemic outcome.

\section{Conclusion}

Preclinical models of cancer generally fail to adequately represent the complexity of human tumor-host interactions. They have revealed, however, some of the potential mechanisms that connect the achievement of radiation-induced tumor control in the breast with an improved breast cancerspecific survival. Preclinical evidence recapitulating the results of the many interactions between radiotherapy and the immune system has inspired ongoing clinical trials of radiotherapy and immunotherapy.

On the other hand, a better understanding of the mechanism of EMT and its implication in cancer metastasis offers an opportunity for expanding the preliminary preclinical data of ionizing radiation effects on residual cancer cells at the tumor cavity.

Figure 2 summarizes the potential effects of ionizing radiation on the surgical cavity, by interfering with EMT as well as with the immune microenvironment at the site of the original tumor.

In conclusion, the existing clinical epidemiological evidence of an association between local control and breast cancerspecific survival has refocused the research community to 


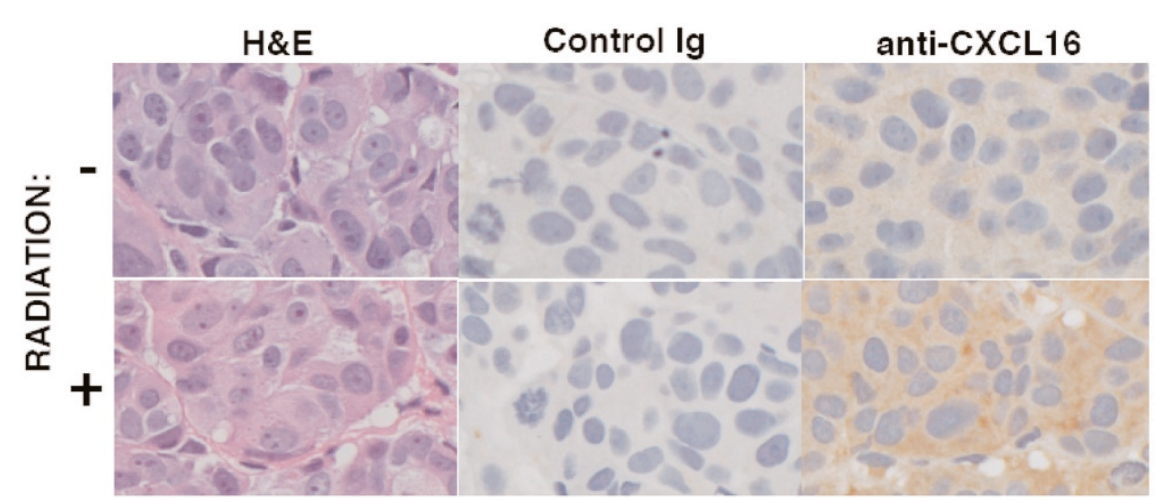

Enhanced expression of the chemokine CXCL16 following irradiation of human breast cancer cells. HTB20 cells, derived from a primary human breast cancer [45], were harvested 48 hours after mock treatment (-) or after irradiation with a dose of 12 Gy $(+)$ and a cell block was prepared as previously described [46]. Sections $(8 \mu \mathrm{m})$ were stained with H\&E or with goat polyclonal antiserum against human CXCL16 (R\&D Systems, Minneapolis, MN, USA) or with normal goat serum as a control, followed by peroxidase-conjugated secondary antibody, were visualized with 3,3diaminobenzidine (DAB Substrate Kit; BD Pharmingen, San Diego, CA, USA), and were counterstained with hematoxylin. Magnification x400.

\section{Figure 2}
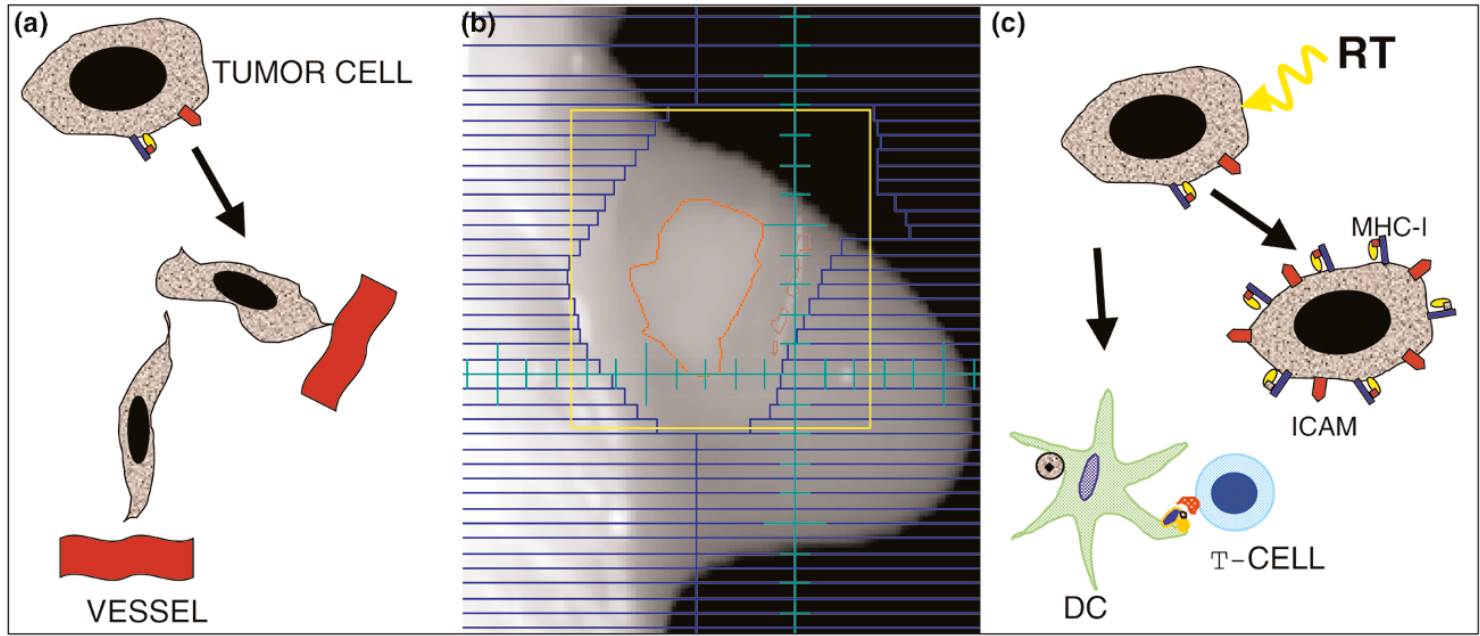

The surgical cavity and its microenvironment. (a) Process of epithelial to mesenchymal transition. (b) Digital radiograph of the lumpectomy cavity targeted by a radiation boost field, limited by a multileaf collimator. Increased density characterizes the persistent fluid at the surgical wound site. (c) Schematic representation of some of the effects radiation has on the immunological microenvironment of the tumor. DC, dendritic cell; ICAM, intracellular adhesion molecule; MHC, major histocompatibility complex; RT, radiation therapy.

study the biologic mechanisms that underlie these findings. From a radiation oncology point of view this is an invaluable opportunity to elucidate novel mechanisms associated with the effects of ionizing radiation, including its unexpected favorable effects on the immune system, a central mediator of the balance between cell survival and death. It is conceivable that successful cooperation between breast cancer radiotherapy and the immune system could result in sustained dormancy, and might reflect on reduced cause-specific mortality.

The organism, after all, routinely refers to the immune system to handle danger signals, with signal transduction pathways that use the same receptors and ligands in response to virus, bacteria or ionizing radiation [37].

\section{Competing interests}

The authors declare that they have no competing interests.

\section{Acknowledgements}

SCF is supported by the Department of Defense Center of Excellence Award BC030282, The Breast Cancer Research Foundation, and Core Grant NIH 5P30CA016087-27. SD is supported by NIH R01 CA113851, Research Scholar award RSG-05-145-01-LIB from the American Cancer Society, and a grant from The Chemotherapy Foundation. 


\section{References}

1. Clarke M, Collins R, Darby S, Davies C, Elphinstone P, Evans E, Godwin J, Gray R, Hicks C, James S, MacKinnon E, McGale P, McHugh T, Peto R, Taylor C, Wang Y ; Early Breast Cancer Trialists' Collaborative Group (EBCTCG): Effects of radiotherapy and of differences in the extent of surgery for early breast cancer on local recurrence and 15-year survival: an overview of the randomised trials. Lancet 2005, 366:2087-2106.

2. Punglia RS, Morrow M, Winer EP, Harris JR: Local therapy and survival in breast cancer. N Engl J Med 2007, 356:2399-2405.

3. Barcellos-Hoff $\mathrm{MH}$ : It takes a tissue to make a tumor: epigenetics, cancer and the microenvironment. J Mammary Gland Biol Neoplasia 2001, 6:213-221.

4. Raven P, Johnson S, Singer S, Losos J: Biology. 7th edition. McGraw-Hill; 2005.

5. MacDonald IC, Groom AC, Chambers AF: Cancer spread and micrometastasis development: quantitative approaches for in vivo models. Bioessays 2002, 24:885-893.

6. Thiery JP: Epithelial-mesenchymal transitions in development and pathologies. Curr Opin Cell Biol 2003, 15:740-746.

7. Yang J, Mani SA, Donaher JL, Ramaswamy S, Itzykson RA, Come C, Savagner P, Gitelman I, Richardson A, Weinberg RA: Twist, a master regulator of morphogenesis, plays an essential role in tumor metastasis. Cell 2004, 117:927-939.

8. Klein CA, Blankenstein TJ, Schmidt-Kittler O, Petronio M, Polzer $\mathrm{B}$, Stoecklein NH, Riethmuller G: Genetic heterogeneity of single disseminated tumour cells in minimal residual cancer. Lancet 2002, 360:683-689.

9. Tagliabue E, Agresti R, Carcangiu ML, Ghirelli C, Morelli D, Campiglio M, Martel M, Giovanazzi R, Greco M, Balsari A, Ménard S: Role of HER2 in wound-induced breast carcinoma proliferation. Lancet 2003, 362:527-533.

10. Coussens LM, Werb Z: Inflammation and cancer. Nature 2002, 420:860-867.

11. Fisher B, Gunduz N, Coyle J, Rudock C, Saffer E: Presence of a growth-stimulating factor in serum following primary tumor removal in mice. Cancer Res 1989, 49:1996-2001.

12. Baum M, Demicheli R, Hrushesky W, Retsky M: Does surgery unfavourably perturb the 'natural history' of early breast cancer by accelerating the appearance of distant metastases? Eur J Cancer 2005, 41:508-515.

13. Nuyten DS, Kreike B, Hart AA, Chi JT, Sneddon JB, Wessels LF, Peterse HJ, Bartelink H, Brown PO, Chang HY, van de Vijver MJ: Predicting a local recurrence after breast-conserving therapy by gene expression profiling. Breast Cancer Res 2006, 8:R62.

14. Murthy BL, Thomson CS, Dodwell D, Shenoy H, Mikeljevic JS, Forman D, Horgan K: Postoperative wound complications and systemic recurrence in breast cancer. $\mathrm{Br} J$ Cancer 2007, 97: 1211-1217

15. Belletti B, Vaidya JS, D'Andrea S, Entschladen F, Roncadin M, Lovat F, Berton S, Perin T, Candiani E, Reccanello S, Veronesi A, Canzonieri V, Trovò MG, Zaenker KS, Colombatti A, Baldassarre G, Massarut S: Targeted intraoperative radiotherapy impairs the stimulation of breast cancer cell proliferation and invasion caused by surgical wounding. Clin Cancer Res 2008, 14:13251332.

16. Farrar JD, Katz KH, Windsor J, Thrush G, Scheuermann RH, Uhr JW, Street NE: Cancer dormancy. VII. A regulatory role for $\mathrm{CD}^{+} \mathrm{T}$ cells and IFN- $\gamma$ in establishing and maintaining the tumor-dormant state. J Immunol 1999, 162:2842-2849.

17. Feuerer M, Rocha M, Bai L, Umansky V, Solomayer EF, Bastert G Diel IJ, Schirrmacher V: Enrichment of memory $T$ cells and other profound immunological changes in the bone marrow from untreated breast cancer patients. Int J Cancer 2001, 92: 96-105.

18. Dunn GP, Bruce AT, Ikeda H, Old LJ, Schreiber RD: Cancer immunoediting: from immunosurveillance to tumor escape. Nat Immunol 2002, 3:991-998.

19. Knutson K, Hailing L, Stone B, Reiman J, Behrens M, Prosperi C, Gad E, Smorlesi A, Disis M: Immunoediting of cancers may lead to epithelial to mesenchymal transition. J Immunol 2006, 177:1526-1533.

20. Lin EY, Nguyen AV, Russell RG, Pollard JW: Colony-stimulating factor 1 promotes progression of mammary tumors to malignancy. J Exp Med 2001, 193:727-740.

21. Leek RD, Lewis CE, Whitehouse R, Greenall M, Clarke J, Harris $\mathrm{AL}$ : Association of macrophage infiltration with angiogenesis and prognosis in invasive breast carcinoma. Cancer Res 1996, 56:4625-4629.

22. Demaria S, Ng B, Devitt ML, Babb JS, Kawashima N, Liebes L Formenti SC: lonizing radiation inhibition of distant untreated tumors (abscopal effect) is immune mediated. Int J Radiat Oncol Biol Phys 2004, 58:862-870.

23. Demaria S, Bhardwaj N, McBride WH, Formenti SC: Combining radiotherapy and immunotherapy: a revived partnership. Int J Radiat Oncol Biol Phys 2005, 63:655-666.

24. Demaria S, Santori FR, Ng B, Liebes L, Formenti SC, Vukmanovic $S$ : Select forms of tumor cell apoptosis induce dendritic cell maturation. J Leukoc Biol 2005, 77:361-368.

25. Demaria S, Kawashima N, Yang AM, Devitt ML, Babb JS, Allison JP, Formenti SC: Immune-mediated inhibition of metastases after treatment with local radiation and CTLA-4 blockade in a mouse model of breast cancer. Clin Cancer Res 2005, 11(2 Pt 1):728-734.

26. Newcomb EW, Demaria S, Lukyanov Y, Shao Y, Schnee T, Kawashima N, Lan L, Dewyngaert JK, Zagzag D, McBride WH, Formenti SC: The combination of ionizing radiation and peripheral vaccination produces long-term survival of mice bearing established invasive GL261 gliomas. Clin Cancer Res 2006, 12:4730-4737.

27. Demaria S, Formenti SC: Sensors of ionizing radiation effects on the immunological microenvironment of cancer. Int J Radiat Biol 2007, 83:819-825.

28. Formenti SC, Dunnington G, Uzieli B, Lenz H, Keren-Rosenberg S, Silberman H, Spicer D, Denk M, Leichman G, Groshen S, Watkins K, Muggia F, Florentine B, Press M, Danenberg K, Danenberg $\mathrm{P}$ : Original p53 status predicts for pathological response in locally advanced breast cancer patients treated preoperatively with continuous infusion 5-fluorouracil and radiation therapy. Int J Radiat Oncol Biol Phys 1997, 39:10591068.

29. Skinner KA, Dunnington G, Silberman H, Florentine B, Spicer D, Formenti SC: Preoperative 5-fluorouracil and radiation therapy for locally advanced breast cancer. Am J Surg 1997, 174:705707; discussion 707-708.

30. Formenti SC, Demaria S: Effects of chemoradiation on tumor-host interactions: the immunologic side. J Clin Oncol 2008, 26:1562-1563; author reply 1563.

31. Formenti SC, Cohen D, Tsao-Wei DD, Muggia FM: Clinical course of locally advanced breast cancer (LABC) patients with pathological response to primary concurrent 5-fluorouracil and radiation (FU/RT). Int J Radiat Oncol Biol Phys 2001, 51: 195-196.

32. Smyth MJ, Godfrey DI, Trapani JA: A fresh look at tumor immunosurveillance and immunotherapy. Nat Immunol 2001, 2:293-299

33. Chakravarty PK, Guha C, Alfieri A, Beri V, Niazova Z, Deb NJ, Fan Z, Thomas EK, Vikram B: Flt3L therapy following localized tumor irradiation generates long-term protective immune response in metastatic lung cancer: its implication in designing a vaccination strategy. Oncology 2006, 70:245-254.

34. Teitz-Tennenbaum S, Li Q, Rynkiewicz S, Ito F, Davis MA, McGinn CJ, Chang AE: Radiotherapy potentiates the therapeutic efficacy of intratumoral dendritic cell administration. Cancer Res 2003, 63:8466-8475.

35. Kim KW, Kim SH, Shin JG, Kim GS, Son YO, Park SW, Kwon BH, Kim DW, Lee CH, Sol MY, Jeong MH, Chung BS, Kang CD: Direct injection of immature dendritic cells into irradiated tumor induces efficient antitumor immunity. Int J Cancer 2004, 109:685-690.

36. Obeid M, Panaretakis T, Joza N, Tufi R, Tesniere A, van Endert P, Zitvogel L, Kroemer G: Calreticulin exposure is required for the immunogenicity of gamma-irradiation and UVC light-induced apoptosis. Cell Death Differ 2007, 14:1848-1850.

37. Apetoh L, Ghiringhelli F, Tesniere A, Obeid M, Ortiz C, Criollo A, Mignot G, Maiuri MC, Ullrich E, Saulnier $P$, Yang $H$, Amigorena $S$, Ryffel B, Barrat FJ, Saftig P, Levi F, Lidereau R, Nogues C, Mira JP, Chompret A, Joulin V, Clavel-Chapelon F, Bourhis J, André F, Delaloge S, Tursz T, Kroemer G, Zitvogel L: Toll-like receptor 4dependent contribution of the immune system to anticancer chemotherapy and radiotherapy. Nat Med 2007, 13:1050-1059.

38. Barcellos-Hoff MH, Park C, Wright EG: Radiation and the microenvironment - tumorigenesis and therapy. Nat Rev Cancer 2005, 5:867-875. 
39. Chakraborty M, Abrams SI, Camphausen K, Liu K, Scott T, Coleman CN, Hodge JW: Irradiation of tumor cells up-regulates Fas and enhances CTL lytic activity and CTL adoptive immunotherapy. J Immunol 2003, 170:6338-6347.

40. Garnett CT, Palena C, Chakraborty M, Tsang KY, Schlom J, Hodge JW: Sublethal irradiation of human tumor cells modulates phenotype resulting in enhanced killing by cytotoxic $T$ lymphocytes. Cancer Res 2004, 64:7985-7994.

41. Reits EA, Hodge JW, Herberts CA, Groothuis TA, Chakraborty M, Wansley EK, Camphausen K, Luiten RM, de Ru AH, Neijssen J, Griekspoor A, Mesman E, Verreck FA, Spits H, Schlom J, van Veelen $\mathrm{P}$, Neefjes JJ: Radiation modulates the peptide repertoire, enhances MHC class I expression, and induces successful antitumor immunotherapy. J Exp Med 2006, 203: 1259-1271.

42. Lugade AA, Sorensen EW, Gerber SA, Moran JP, Frelinger JG, Lord EM: Radiation-induced IFN- $\gamma$ production within the tumor microenvironment influences antitumor immunity. J Immunol 2008, 180:3132-3139.

43. Matsumura S, Wang B, Kawashima N, Braunstein S, Badura M, Cameron TO, Babb JS, Schneider RJ, Formenti SC, Dustin ML, Demaria S: Radiation-induced CXCL16 release by breast cancer cells attracts effector T cells. $J$ Immunol 2008, 181: 3099-3107.

44. Biswas S, Guix M, Rinehart C, Dugger TC, Chytil A, Moses HL, Freeman ML, Arteaga CL: Inhibition of TGF- $\beta$ with neutralizing antibodies prevents radiation-induced acceleration of metastatic cancer progression. J Clin Invest 2007, 117:13051313.

45. Lasfargues EY, Coutinho WG, Redfield ES: Isolation of two human tumor epithelial cell lines from solid breast carcinomas. J Natl Cancer Inst 1978, 61:967-978.

46. Yang GC, Wan LS, Papellas J, Waisman J: Compact cell blocks. Use for body fluids, fine needle aspirations and endometrial brush biopsies. Acta Cytol 1998, 42:703-706. 